\title{
Verwahrung gefährlicher Straftäter: Kluft zwischen politischen Forderungen und medizinisch-wissenschaftlicher Machbarkeit
}

\author{
Dr. med. Gerhard Ebner ${ }^{a}$, Prof. Dr. med. Volker Dittmann ${ }^{b}$, Dr. med. Ursula Steiner-König ${ }^{c}$, Dr. med. Hans Kurt ${ }^{d}$
}

a Leiter Ressort Kommunikation und Vernehmlassungen SGPP, Präsident Schweizerische Vereinigung Psychiatrischer Chefärzte, Chefarzt und Vorsitzender der Geschäftsleitung Psychiatrische Dienste Schaffhausen

b Ordinarius für Forensische Psychiatrie und Rechtsmedizin an der Universität Basel

c Vizepräsidentin FMH

d Präsident Schweizerische Gesellschaft für Psychiatrie und Psychotherapie

\section{Korrespondenz: \\ SGPP-Sekretariat \\ Postgasse 17 \\ Postfach 686 \\ CH-3000 Bern 8 \\ Tel. 0313138833 \\ Fax 0313138899}

E-Mail: sgpp@psychiatrie.ch

Internet: www. psychiatrie.ch
Die psychiatrischen Fachgesellschaften (SGPP, SVPC, Fachvertreter Psychiatrie) haben zusammen mit der FMH einen offenen Brief an Bundesrat Christoph Blocher geschrieben. Wir machen damit auf unüberwindbare Schwierigkeiten bei der Umsetzung des Verfassungsartikels 123a (über die lebenslängliche Verwahrung extrem gefährlicher Straftäter) aufmerksam. Ferner bieten wir Lösungsansätze an.

Wir können keine gültigen psychiatrischen Prognosen für unbestimmte Zeiträume («lebenslang») stellen

Schon jetzt müssen wir bei etwa einem Drittel der Begutachteten damit rechnen, sie fälschlicherweise als extrem gefährlich und untherapierbar anzusehen. Mit zunehmendem Prognosezeitraum - ähnlich wie bei der Wetterprognose sinkt die Gültigkeit der Vorhersage. Aus wissenschaftlicher Sicht ist das Stellen einer lebenslangen Prognose mit Ausnahme von wenigen Extremfällen nicht verantwortbar.

Neue wissenschaftliche Erkenntnisse, die eine Neubegutachtung ermöglichen würden, sind schwer zu erlangen

Therapiestudien, die neue wissenschaftliche Erkenntnisse erwarten lassen, sind unrealistisch: wie sollen bei lebenslänglich Verwahrten, die als gefährlich und untherapierbar gelten, Kontrollgruppen gebildet werden, die in Freiheit die Wirksamkeit dieser Therapiemethoden nachweisen?

\section{Wir haben nicht genügend qualifizierte Gutachter}

Das Gesetz sieht vor, zwei unabhängige, gut ausgebildete und erfahrene Gutachter für diese Gutachten zu finden. Sie sollten nicht in die Behandlung involviert sein, nicht in der gleichen Institution arbeiten und auch nicht Mitglied als Experten der eidgenössischen Kommission sein, zurzeit eine unrealistische Forderung.
Ohne eine entsprechende Anlasstat können wir nur allein anhand von Persönlichkeitsmerkmalen keine Prognose zur Gefährlichkeit abgeben

Die nachträgliche Anordnung einer Verwahrung bedarf weder einer bestimmten strafrechtlichen Vorbelastung des Täters noch einer Anlasstat bestimmter Schwere. Bei der Gefährlichkeitsprognose, die der Gutachter erstellt, hat dieser dann unter anderem anhand von Persönlichkeitsmerkmalen zu beurteilen, ob schwere Delikte in $\mathrm{Zu}$ kunft zu erwarten sind. Dabei nimmt aber die Analyse der begangenen Taten einen sehr hohen Stellenwert ein, wie auch die Frage, ob und wie sich der Täter in der Folge mit den begangenen Taten auseinandersetzt. Die Verlagerung der Begutachtung einzig auf die Ebene möglicher künftiger Delikte ohne Eingrenzung auf ein bereits stattgefundenes Delikt verunmöglicht eine seriöse Prognoseerstellung. Die Fehlerquote wäre viel zu hoch, eine gesellschaftlich kaum verantwortbare Zahl von Menschen, die niemandem etwas zuleide tun würden, müssten verwahrt werden. Hier schlagen wir die Streichung dieses Artikels vor oder müssen die Beurteilung dem Richter überlassen. Mit einer seriösen Gutachtenerstellung hat diese Forderung nichts mehr zu tun.

\section{Welche Massnahmen schlagen wir vor?}

Die Fachgesellschaften haben Massnahmen in die Wege geleitet, um die Ausbildung der Gutachter zu verbessern, für mehr gut ausgebildete Gutachter zu sorgen und die Gerichte zu befähigen, die Qualität der Gutachten besser beurteilen zu können. Ein entsprechendes Grundlagenwerk für Juristen und Medizin wird demnächst erscheinen (Ebner et al. 2005: Psychiatrie und Recht).

Zur Verbesserung der Gültigkeit von Prognosen schlagen wir verstärkte Forschungsanstrengungen vor, um die Grundlagen für eine seriöse Begutachtung zu verbessern.

Selbstverständlich sind wir der Bundesverfassung und damit dem Willen des Souveräns verpflichtet. Niemand kann uns aber zwingen, etwas zu tun, das wir nicht tun können. 